
ISSN 1813-548X

\title{
Typologie physico-chimique et métallique des eaux du fleuve Sénégal au niveau de la ville de Rosso (Mauritanie)
}

\section{Eby OULD MOHAMEDOU $13^{*}$, Ahmed LEBKIRI ${ }^{1}$, El Housseine RIFI', Mustapha LEBKIRI ${ }^{1}$, Mohamed FADLI ${ }^{2}$, Maxime PONTIE ${ }^{3}$, Abdel Kader OULD MAHMOUD ${ }^{1}$ et Mohamed Lemine FAGEL4}

'Laboratoire de chimie Organique et Procédés d'Extraction, Faculté des Sciences, université Ibn Tofail, Kenitra, Maroc.

2 Laboratoire de Biodiversité et Ressources Naturelles, Faculté des

Sciences, université Ibn Tofail, Kenitra, Maroc.

${ }^{3}$ GEPEA, UMR- CNRS 6144, Université d'Angers, 2 Bd Lavoisier 49045

Angers cedex 01, France.

${ }^{4}$ Centre de Recherche Appliquée aux Energies Renouvelables (CRAER),

Faculté des Sciences et Techniques, Université de Nouakchott, Mauritanie.

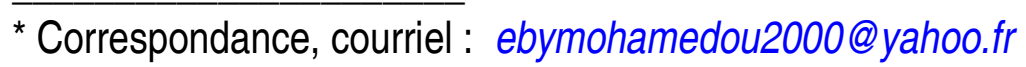

\section{Résumé}

L'étude concerne une analyse de la typologie physico-chimiques et métalliques des eaux du fleuve Sénégal: Cas du delta Mauritanien localisé dans la ville de Rosso. L'évaluation de 19 variables est effectuée par dosage volumétrique et spectroscopique (spectroscopie d'absorption atomique Flamme et Four, ICP) selon le Rodier au cours de la période d'étude dans les deux saisons de l'année 2006: Saison froide "Septembre" et saison sèche "Mars". Les résultats montrent que :

- Parmi 19 variables physicochimiques et métalliques évalués seules 14 déterminent la typologie physicochimique du milieu étudié.

- Deux groupements de relevées sont différenciés. Le premier rassemble des relevés tous effectués en été et caractérisés par des concertations élevés en $\mathrm{Ca}^{2+}, \mathrm{Mg}^{2+}, \mathrm{Na}^{+}, \mathrm{Zn}^{2+}$ et faibles en $\mathrm{NO}^{3-}$ et $\mathrm{K}^{+}$. Le second 
groupement est formé par des relevés hivernaux présentant des caractéristiques physicochimiques opposées à celles des relevés du premier groupement. L'effet du facteur " saison » est donc bien dégagé. Pour les teneurs en $\mathrm{Al}^{3+}$ et le $\mathrm{Sr}^{2+}$ le facteur saison n'intervient pas. En outre, l'activité domestique et les rejets des eaux usées favorisent la pollution organique de certaines parties de la zone prospectée alors que les rejets des eaux usées industrielles de la sucrerie sénégalaise C.S.S. polluent en $\mathrm{Al}^{2+}$ et $\mathrm{Sr}^{2+}$ d'autres stations. Mais, d'une manière générale les teneurs des éléments chimiques évalués sont dans les nomes de l'eau d'irrigation.

Mots-clés : eau potable, eau usée, pollution, concentration métallique, paramètres physico-chimiques, fleuve Sénégal, Mauritanie

\section{Abstract}

\section{Physico-chemical and metal typology of Senegal river's water at Rosso city in Mauritania}

The study concerns the determination of the quality and type physicochemical and metal waters of the Senegal River: the case of delta Mauritanien located in the town of Rosso. The assessment of variables studied was done by volumetric and spectroscopic assay (atomic absorption spectroscopy and flame Four, according to Rodier ICP during the study period in both seasons of the year 2006: Cold Season"in September dry season"and"March". The results show that: - Among 19 variables evaluated physicochemical and metal only 14 chemicophysical determine the type of environment studied. - Two groups are identified differentiated. The first includes all statements made in the summer and have dialogues high $\mathrm{Ca}^{2+}, \mathrm{Mg}^{2+}, \mathrm{Na}^{+}, \mathrm{Zn}^{2+}$ and low in $\mathrm{NO}^{3}$-and $\mathrm{K}^{+}$. The second grouping is formed by winter surveys showing physicochemical characteristics opposite to those of in the first group the effect of the factor 'season' is well earned. For contents of $\mathrm{Al}{ }^{3+}$ and $\mathrm{Sr}^{2+}$ season factor is irrelevant. The activity and domestic 
wastewater discharges promote organic pollution in some parts of the area surveyed, while discharges of industrial wastewater of the sugar Sénégalaise CSS pollute in $\mathrm{Al}^{3}+$ and $\mathrm{Sr}^{2}+$ other stations. But, in general the levels of elements are evaluated in the prefectures of irrigation water.

Keywords : drinking water, sloppy water, pollution, metallic concentration, physico-chemical parameters, Senegal river, Mauritania.

\section{Introduction}

L'importance de l'eau dans l'économie humaine ne cesse de croître. Sous la pression des besoins considérables de la civilisation moderne, on est passé de l'emploi des eaux de sources et de nappes, à une utilisation de plus en plus accrue des eaux de surface. Ainsi, l'approvisionnement en eau douce devient de plus en plus difficile tant en raison du développement accéléré des techniques industrielles modernes que de l'accroissement de la population et de son amélioration de son niveau de vie [1].

Notons que le problème de la quantité et de la qualité des eaux nécessaires à la population et à son développement économique se pose pour de nombreux pays notamment ceux qui ne reçoivent que peu de précipitations tels que les pays du Sahel. La Mauritanie, par exemple, à cause de son climat à caractère sahélien, est un pays déficitaire en eau. En effet, la faible pluviométrie, l'importance de l'évaporation ainsi que la nature géologique du pays font que la Mauritanie est pauvre en ressources hydriques. Les ressources sont donc limitées alors que les besoins augmentent. En outre, c'est le fleuve Sénégal qui joue un rôle très important comme source principale d'eau de surface pour la Mauritanie, le Sénégal et le Mali. Les eaux de ce fleuve sont susceptibles d'être utilisées comme eaux potables et eaux d'irrigation. En effet, ce fleuve arrose les quatre pays et draine un bassin versant de $340000 \mathrm{~km}^{2}$ [2]. II est alimenté par trois affluents principaux : le Bafing, le Bakoye et la Falamé qui prennent tous leurs sources dans le massif du Fouta Djalon en Guinée et qui se regroupent pour former ce fleuve qui d'une longueur 
de $1800 \mathrm{~km}$ traverse les quatre pays citées pour aboutir son embouchure juste après le barrage de Diama en Mauritanie [3].

Actuellement, ce fleuve vital subit des contraintes qui menacent ses qualités physiques, chimiques et métalliques. A différents points géographiques ce cours d'eau reçoit des rejets d'eau usée domestique ou industrielle. Sachant que la pollution de l'eau est comme étant toute modification des propriétés physiques, chimiques ou biologiques, ou tout rejet de substances liquides, gazeuses ou solides dans l'eau de façon à créer une nuisance ou à rendre cette eau dangereuse ou préjudiciable du point de vue, soit de la santé, de la sécurité et du bien être publique, soit de ses usages destinés à des fins domestiques, commerciales, industrielles, agricoles, récréatives et autres, soit de la faune sauvage et aquatique [4], nous avons vu nécessaire d'estimer la qualité et la typologie physico-chimique des eaux du fleuve Sénégal au niveau du delta de la ville de Rosso et d'établir une typologie physico-chimique et métallique du milieu à ce niveau.

\section{Matériel et méthodes}

\section{2-1. Stations d'études}

Afin de contribuer à l'évaluation de la qualité des eaux sur notre environnement et de pouvoir envisager des solutions fiables et durables, nous avons choisi comme site d'étude le delta du fleuve Sénégal (Rosso) et cela pour plusieurs raisons. Le delta avoisine plusieurs agglomérations humaines (Keur, Mesein, Rosso Sénégal, Rosso Mauritanie,...) et une unité industrielle, Compagnie Sucrière Sénégalaise (C.S.S). Le milieu étudié reçoit des rejets domestiques et rejets industriels. Le risque de la contamination du Fleuve et de la nappe phréatique qui alimente toute la région en eau potable est potentielle. Ainsi, il d'une nécessité d'évaluer les caractéristiques physico-chimiques et métallique des eaux du fleuve Sénégal. Pour atteindre cet objectif, nous avons choisi quatre stations de prélèvement, situées sur l'axe du fleuve (Figure 1), dont trois sont en 
Mauritanie et une au Sénégal. Celles-ci ont été choisies selon leur accessibilité, leur proximité d'éventuelles sources de pollution et leur répartition géographique est comme suite :

- La Station dite Moyen de Transport (ST) : située au Sud-Ouest de Rosso près du Bag assurant le lien de transport entre la Mauritanie et le Sénégal.

- La Station d'Agriculture (SA): Située au Sud-Ouest de la ville de Rosso, à $7 \mathrm{~km}$ en aval de la satation ST.

- La Station Domestique (SD) : située à $3 \mathrm{Km}$ en amont de ST et juste dans la zone des rejets des eaux usées et où l'activité humaine est très importante (lavage du linge de vaisselle, etc.)

- La Station de Point de Rejet (SPR) : située, à $10 \mathrm{k}$ en amont de SD et en aval des rejet des eaux usées de la C.S.S. Cette station a été subdivisée en deux sous-stations : $\mathbf{S R v}$ située à $5 \mathrm{~m}$ après le point de rejet et $\mathbf{S R m}$ à $5 \mathrm{~m}$ en avant du point de rejet.

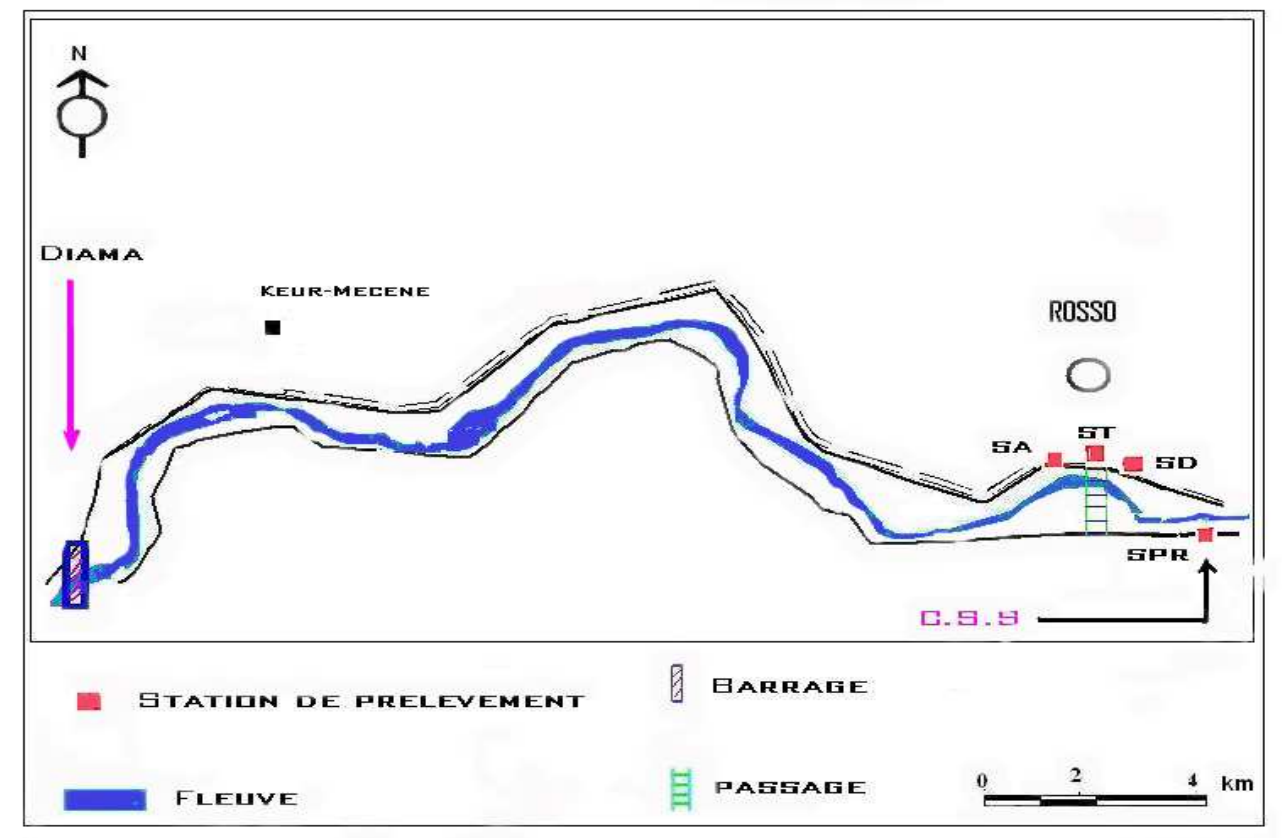

Figure 1 : Localisation des sites de prélèvement 
Pour ce faire, nous avons estimé, en été et en hiver, 19 variables qui caractérisent le milieu prospecté (Tableau 1).

Tableau 1 : Codes employés pour les paramètres physicochimiques et métalliques évalués

\begin{tabular}{|l|c|c|c|}
\hline Variables & Codes & Variables & Codes \\
\hline Température & $\mathrm{T}$ & Sodium & $\mathrm{Na}^{+}$ \\
\hline $\begin{array}{l}\text { Potentiel } \\
\text { hydrogène }\end{array}$ & $\mathrm{pH}$ & Potassium & $\mathrm{K}^{+}$ \\
\hline $\begin{array}{l}\text { Conductivité } \\
\text { électrique }\end{array}$ & Cond & Dioxyde de carbone & $\mathrm{CO}_{2}$ \\
\hline $\begin{array}{l}\text { Matières } \\
\text { suspension }\end{array}$ & $\mathrm{M.E.S}_{3}$ & $\mathrm{Fer}$ & $\mathrm{Fe}^{2+}$ \\
\hline Nitrates & $\mathrm{NO}_{3}{ }^{--}$ & Zenc & $\mathrm{Zn}^{2+}$ \\
\hline Sulfates & $\mathrm{SO}_{4}{ }^{--}$ & Aluminum & $\mathrm{Al}^{3+}$ \\
\hline Bicarbonates & $\mathrm{HCO}_{3}{ }^{-}$ & Orthophosphates & $\mathrm{PO}_{4}{ }^{2-}$ \\
\hline Chlorure & $\mathrm{Cl}^{-}$ & Surinum & $\mathrm{Sr}^{2+}$ \\
\hline Calcium & $\mathrm{Ca}^{2+}$ & Nitrites & $\mathrm{NO}_{2}^{-}$ \\
\hline Magnesium & $\mathrm{Mg}^{2+}$ & - & - \\
\hline
\end{tabular}

\section{2-2. Echantillonnage}

Les prélèvements des eaux à analyser ont été effectués dans des bouteilles en plastique dans des zones où l'eau n'est pas stagnante [5].

\section{2-3. Méthode d'analyse}

Au même moment du prélèvement des échantillons d'eau, certaines mesures physico-chimiques de l'eau on été effectués in situ : température de l'eau, conductivité électrique et $\mathrm{pH}$. Le reste des évaluations a été réalisé par dosage volumétrique ou dosages spectroscopique, selon les méthodes d'analyse proposées par Rodier [6], dans le Laboratoire de I'Institut National de Recherches et Santé Publique en Mauritanie et le Laboratoire d'Analyses Physico-chimiques de l'ONHYM de Rabat, Maroc [7]. 
Par ailleurs, dans le but de visualiser et d'analyser les corrélations existantes entre les différentes variables à travers leurs structurations et leurs orientations, d'identifier les principaux facteurs responsables de la qualité des eaux de l'environnement prospecté, nous avons statistiquement traité l'ensemble des données par l'Analyse en Composantes Principales (A.C.P). Cette technique permet de déterminer un système d'axes de référence hiérarchisés tout en diminuant le nombre des dimensions de l'espace dans lequel on projette les points-observations. Elle est utile pour l'analyse des données quantitatives se présentant sous la forme de tableau à $\mathrm{N}$ observations et $P$ variables $[8,9]$.

De même, pour faciliter la représentation graphique des résultats d'analyse, nous avons numéroté les prélèvements de la manière illustrée par le (Tableau 2) :

Tableau 2: Numéros des prélèvements réalisés dans les cinq stations pendant les deux saisons de l'année (Crue, et étiage) Rosso 2006

\begin{tabular}{|l|c|c|}
\hline Stations & Eté (03/06) & Hiver (09/06) \\
\hline ST & 1 & 6 \\
\hline SA & 2 & 7 \\
\hline SD & 3 & 8 \\
\hline SRv & 4 & 9 \\
\hline SRm & 5 & 10 \\
\hline
\end{tabular}

\section{2-4. Elaboration de la matrice}

Durant deux saisons, l'état hydrochimique du fleuve est appréhendé à partir des mesures de 19 variables physicochimiques et métalliques. L'ensemble des données est regroupé sous forme d'une matrice constituée par un tableau à double entrée de type (19 variables x 10 prélèvements), (Tableau 3). 
Tableau 3 : Variation des paramètres physico-chimiques et métalliques des eaux du fleuve Sénégal, Rosso 2006 suite

\begin{tabular}{lccc}
\hline Les axes & C1 & C2 & C3 \\
\hline Valeurs propres & 9,08 & 3,80 & 2,21 \\
\% variance & 47,80 & 20,02 & 11,65 \\
\% cumulée & 47,80 & 67,82 & 79,48 \\
\hline
\end{tabular}

\section{Résultats}

L'analyse des résultats permet de constater que la majeure partie des informations est expliquée par les trois premiers axes factoriels (Tableau 4). Les contributions des différents paramètres dans la formation des trois premiers axes factoriels $\mathrm{C} 1, \mathrm{C} 2$ et $\mathrm{C} 3$ sont respectivement de $47,80 \%$, $20,02 \%$ et $11,65 \%$ soit un totale de $79,47 \%$ de l'information expliquée. Par suite, le maximum de l'inertie totale est cumulé par les plans formés par les axes factoriels $\mathrm{C} 1 \times \mathrm{C} 2$ et $\mathrm{C} 1 \times \mathrm{C} 3$. Ainsi, la signification physicochimique et métallique des axes factoriels $\mathrm{C} 1, \mathrm{C} 2$ et $\mathrm{C} 3$ sont nécessaires.

Tableau 4 : Valeurs propres et pourcentages de contribution des trois premières composantes

\begin{tabular}{|c|c|c|c|c|c|c|c|c|cc|}
\hline $\begin{array}{c}\mathbf{N}^{\circ} \\
\text { des } \\
\text { relevés }\end{array}$ & $\mathbf{M g}^{2+}$ & $\mathbf{N a}^{+}$ & $\mathbf{K}^{+}$ & $\mathbf{C O}_{\mathbf{2}}$ & $\mathbf{P O}_{\mathbf{4}}{ }^{2-}$ & $\mathbf{N O}_{\mathbf{2}}{ }^{-}$ & $\mathbf{F e}^{2+}$ & $\mathbf{Z n}^{2+}$ & $\mathbf{A ~}^{3+}$ & $\mathbf{S r}^{2+}$ \\
\hline $\mathbf{1}$ & 1,7 & 13 & 3 & 20,2 & 0,25 & 0,75 & 0,108 & 0,078 & 0,046 & 0,026 \\
\hline $\mathbf{2}$ & 2,6 & 11 & 4 & 17,2 & 0,36 & 1,1 & 0,078 & 0,108 & 0,054 & 0,074 \\
\hline $\mathbf{3}$ & 2,32 & 15 & 5 & 23,5 & 0,123 & 0,235 & 0,098 & 0,092 & 0,122 & 0,18 \\
\hline $\mathbf{4}$ & 2,65 & 12 & 5 & 15,35 & 0,32 & 0,05 & 0,056 & 0,099 & 0,105 & 0,1 \\
\hline $\mathbf{5}$ & 2,54 & 10 & 3 & 24 & 0,105 & 0,15 & 0,073 & 0,085 & 0,071 & 0,031 \\
\hline $\mathbf{6}$ & 0,56 & 9 & 7 & 22,6 & 0,23 & 0,76 & 0,102 & 0,071 & 0,062 & 0,086 \\
\hline $\mathbf{7}$ & 1,35 & 8 & 6 & 12,65 & 0,215 & 1,05 & 0,114 & 0,069 & 0,059 & 0,037 \\
\hline $\mathbf{8}$ & 0,95 & 7 & 10 & 19 & 0,405 & 0,98 & 0,121 & 0,0605 & 0,098 & 0,013 \\
\hline $\mathbf{9}$ & 0,89 & 9 & 8 & 28,45 & 0,623 & 0,35 & 0,152 & 0,052 & 0,093 & 0,014 \\
\hline $\mathbf{1 0}$ & 1,15 & 6 & 9 & 26,5 & 0,518 & 0,45 & 0,195 & 0,047 & 0,057 & 0,022 \\
\hline
\end{tabular}




\section{3-1. Signification des axes de l'A.C.P}

Le degré de contribution de 19 variables physico-chimiques et métalliques dans l'inertie des axes factoriels $\mathrm{C} 1, \mathrm{C} 2$ et $\mathrm{C} 3$ est enregistré par le (Tableau 5).

Tableau 5: Pourcentage de contribution de 19 variables physicochimiques et métalliques dans la constitution des trois principaux axes factoriels

\begin{tabular}{|c|c|c|c|}
\hline Variables & C1 & C2 & C3 \\
\hline$T$ & 6,274 & 5,490 & 1,356 \\
\hline $\mathrm{pH}$ & 1,269 & 11,563 & 12,030 \\
\hline Cond & 2,326 & $\underline{13,673}$ & $\overline{0,364}$ \\
\hline M.E.S & 5,189 & $\overline{4,160}$ & 4,115 \\
\hline $\mathrm{NO}_{3}^{-}$ & $\underline{8,184}$ & 0,525 & 0,299 \\
\hline $\mathrm{SO}_{4}^{2-}$ & 4,799 & 1,076 & $\underline{12,105}$ \\
\hline $\mathrm{HCO}_{3}-$ & 6,034 & 1,786 & $\overline{3,513}$ \\
\hline $\mathrm{Cl}^{-}$ & 6,124 & 3,047 & 1,233 \\
\hline $\mathrm{Ca}^{2+}$ & 9,940 & 0,145 & 0,000 \\
\hline $\mathrm{Mg}^{2+}$ & 8,526 & 0,099 & 0,058 \\
\hline $\mathrm{Na}^{+}$ & 7,757 & 0,384 & 5,590 \\
\hline $\mathrm{K}^{+}$ & 7,277 & 6,310 & 1,140 \\
\hline $\mathrm{CO}_{2}$ & 1,746 & 7,158 & 22,014 \\
\hline $\mathrm{Fe}^{2+}$ & 5,929 & 2,063 & 8,400 \\
\hline $\mathrm{Zn}^{2+}$ & $\underline{8,122}$ & 1,906 & $\overline{3,095}$ \\
\hline $\mathrm{Al}^{3+}$ & $\overline{0,991}$ & $\underline{17,111}$ & 6,314 \\
\hline $\mathrm{PO}_{4}{ }^{2-}$ & 3,619 & $\overline{5,931}$ & 3,150 \\
\hline $\mathrm{Sr}^{2+}$ & 3,523 & $\underline{9,425}$ & 5,275 \\
\hline $\mathrm{NO}_{2}^{-}$ & 2,372 & $\overline{8,148}$ & $\underline{9,950}$ \\
\hline
\end{tabular}

\section{3-2. Axes factoriels $\mathrm{C} 1, \mathrm{C} 2$ et $\mathrm{C} 3$}

Rappelons que deux variables proches sur le graphique n'ont pas obligatoirement les comportements voisins et ce en raison de la projection qui peut parfois être non fiable. II est donc conseillé de voir l'importance de cosinus carrés de l'angle formé par le vecteur de ce point et le plan de projection de la variable pour évaluer la qualité de la projection de cette variable sur cet tel ou tel axe factoriel. Le (Tableau 6) montre plus le cosinus carrée est élevé plus la variable est liée à l'axe 
factoriel. A l'inverse, plus le cosinus carrés est proche de 0 (zéro) moins la variable est liée à cet axe.

Tableau 6 : Cosinus carrés des variables

\begin{tabular}{lccc}
\hline & $\mathbf{C 1}$ & $\mathbf{C 2}$ & $\mathbf{C 3}$ \\
\hline $\mathbf{T}$ & $\mathbf{0 , 5 7 0}$ & 0,209 & 0,030 \\
$\mathrm{pH}$ & 0,115 & $\mathbf{0 , 4 4 0}$ & 0,266 \\
$\mathrm{Cond}$ & 0,211 & $\mathbf{0 , 5 2 0}$ & 0,008 \\
$\mathrm{M.E.S}$ & $\mathbf{0 , 4 7 1}$ & 0,158 & 0,091 \\
$\mathrm{NO}_{3}{ }^{-}$ & $\mathbf{0 , 7 4 3}$ & 0,020 & 0,007 \\
$\mathrm{SO}_{4}{ }^{2-}$ & $\mathbf{0 , 4 3 6}$ & 0,041 & 0,268 \\
$\mathrm{HCO}_{3}{ }^{-}$ & $\mathbf{0 , 5 4 8}$ & 0,068 & 0,078 \\
$\mathrm{Cl}^{-}$ & $\mathbf{0 , 5 5 6}$ & 0,116 & 0,027 \\
$\mathrm{Ca}^{2+}$ & $\mathbf{0 , 9 0 3}$ & 0,006 & 0,000 \\
$\mathrm{Mg}^{2+}$ & $\mathbf{0 , 7 7 4}$ & 0,004 & 0,001 \\
$\mathrm{Na}^{+}$ & $\mathbf{0 , 7 0 5}$ & 0,015 & 0,124 \\
$\mathrm{~K}^{+}$ & $\mathbf{0 , 6 6 1}$ & 0,240 & 0,025 \\
$\mathrm{CO}_{2}$ & 0,159 & 0,272 & 0,488 \\
$\mathrm{Fe}^{2+}$ & $\mathbf{0 , 5 3 9}$ & 0,078 & 0,186 \\
$\mathrm{Zn}^{2+}$ & $\mathbf{0 , 7 3 8}$ & 0,073 & 0,069 \\
$\mathrm{Al}^{3+}$ & 0,090 & $\mathbf{0 , 6 5 1}$ & 0,140 \\
$\mathrm{PO}_{4}{ }^{2-}$ & $\mathbf{0 , 3 2 9}$ & 0,226 & 0,070 \\
$\mathrm{Sr}^{2+}$ & 0,320 & $\mathbf{0 , 3 5 9}$ & 0,117 \\
$\mathrm{NO}_{2}{ }^{-}$ & 0,215 & $\mathbf{0 , 3 1 0}$ & 0,220 \\
\hline
\end{tabular}

L'analyse des corrélations entre les variables et les axes factoriels révèle la nature de ces axes. Le Tableau 6 montre que les variables étudiées ne sont pas toutes bien représentées sur le cercle des corrélations; certaines variables sont présentent des cosinus carrés trop faibles par rapport à l'unité ; en conséquence, ils ne sont pas bien représentés dans les cercles de projection d'où elles ne doivent pas prendre beaucoup d'espace dans l'explication de l'axe factoriel concerné. On en cite, le $\mathrm{pH}$, $\mathrm{NO}_{2}{ }^{-}, \mathrm{Al}^{3+}$, Cond, $\mathrm{Sr}^{2+}$ et $\mathrm{CO}_{2}$.

En revanche, les variables qui contribuent d'une manière principale dans la constitution de l'axe $\mathrm{C} 1$ sont le $\mathrm{Ca}^{2+}, \mathrm{Mg}^{2+}, \mathrm{Na}^{+}, \mathrm{Zn}^{2+}, \mathrm{NO}^{3-}$ et $\mathrm{K}^{+}$. Du coté positif vers le coté négatif (Figure 2) cette axe présente un gradient 
de concentration croissant pour $\mathrm{Ca}^{2+}, \mathrm{Mg}^{2+}, \mathrm{Na}^{+}$et décroissent pour $\mathrm{Zn}^{2+}$, $\mathrm{NO}_{3}$ - et $\mathrm{K}^{+}$. L'axe $\mathrm{C} 1$ correspond aussi à gradient de salinité représentant les teneurs du $\mathrm{Na}^{+}$et $\mathrm{K}^{+}$du milieu. Notons que la majorité des paramètres signalés participent activement dans le degré de minéralisation des eaux du milieu.

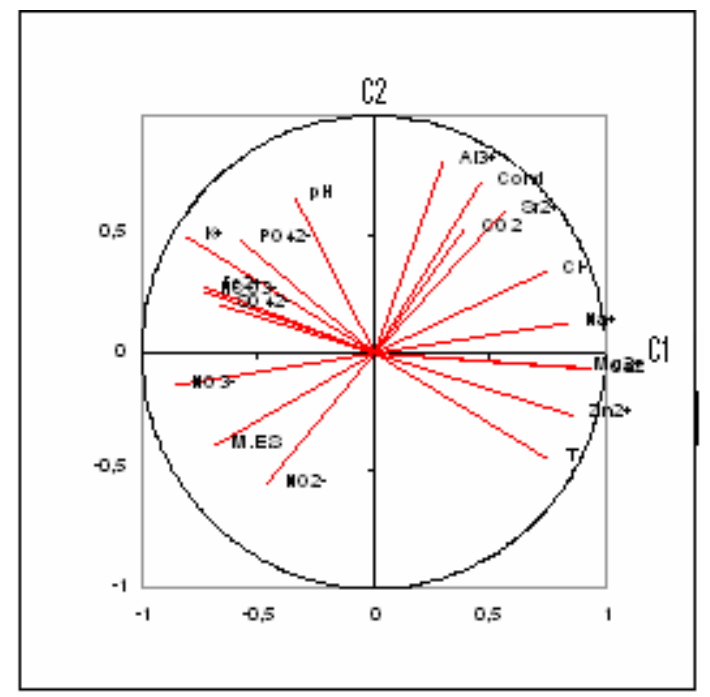

Figure 2 : Cercle de corrélation des différentes variables sur le plan factoriel $\mathrm{C} 1$ et $\mathrm{C} 2$

Concenant l'axe C2, trois variables, toutes situées de sont coté positif, contribuent remarquablement à sa constitution : Cond, $\mathrm{Al}^{3+} \mathrm{et} \mathrm{Sr}^{2+}$. Ainsi, vis-à-vis des deux métaux $\mathrm{Al}^{3+}$ et $\mathrm{Sr}^{2+}$, l'axe $\mathrm{C} 2$ représente un gradient de pollution métallique, croisent de coté négatif vers le coté positif de l'axe.

En outre, selon le Tableau 5, cinq variables participent activement dans la constitution de l'axe $\mathrm{C} 3$ à savoir $\mathrm{pH}, \mathrm{SO}_{4}^{2-}, \mathrm{NO}_{2}-\mathrm{CO}_{2}$ et $\mathrm{Fe}^{2+}$. $\mathrm{A}$ l'exception du fer, cet axe correspond à un gradient de pollution organique. De même, seule le $\mathrm{CO}_{2}$ est située sur le coté positif de l'axe cité (Figure 3). 


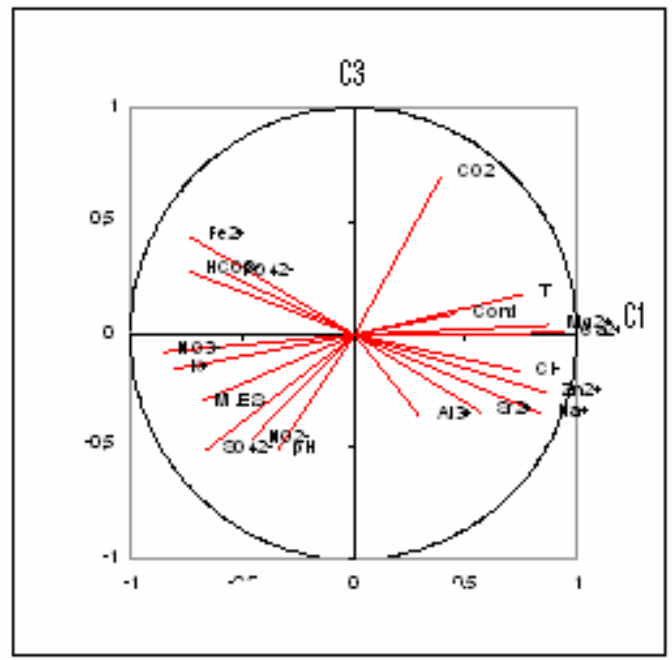

Figure 3 : Cercle de corrélation des différentes variables sur le plan factoriel $\mathrm{C} 1$ et $\mathrm{C3}$

En conclusion on déduit que parmi les 19 variables étudiés seules 14 participent dans la constitution des axes $\mathrm{C} 1, \mathrm{C} 2$ et $\mathrm{C} 3$. Les cinq variables qui restent à savoir $\mathrm{T}, \mathrm{MES}, \mathrm{HCO}_{3}{ }^{-}, \mathrm{Cl}$ et $\mathrm{PO}_{4}^{2-}$ 'ont pas donc une part importante dans la détermination de la typologie physico-chimique et métallique du milieu étudié.

\section{4. Analyse des plans factoriels $\mathrm{C} 1 \times \mathrm{C} 2$ et $\mathrm{C} 1 \times \mathrm{C} 3$}

L'analyse de la carte factorielle $\mathrm{C} 1 \times \mathrm{C} 2$ (Figure 4) permet de distinguer deux groupements de relevées. Le premier groupement $(E)$, situé dans le coté négatif dans l'axe $C 1$, rassemble des relevés $(1,2,3,4$ et 5$)$ tous effectués en été; en situation opposée, un second groupement formé par les relevés hivernaux $(6,7,8,9$ et 10). L'effet du facteur " saison " est donc bien dégagé.

De même, si on tient compte de la signification physicochimique de l'axe $C 1$ on déduit que les relevés du groupement $E$, sont caractérisés par des concertations élevés en $\mathrm{Ca}^{2+}, \mathrm{Mg}^{2+}, \mathrm{Na}^{+}, \mathrm{Zn}^{2+}$ et faibles en $\mathrm{NO}_{3}{ }^{-}$et $\mathrm{K}^{+}$. En outre, la signification de l'axe $\mathrm{C} 2$ nous a permit de noter l'existence de relevés ayant des concentrations élevées en $\mathrm{Al}^{3+}$ et faible en $\mathrm{Sr}^{2+}$ et une 
conductivité élevée tels que les relevés $3,4,8$ et 9 et d'autres relevés à caractéristiques opposées tels que numérotés 1,2 ou 7.

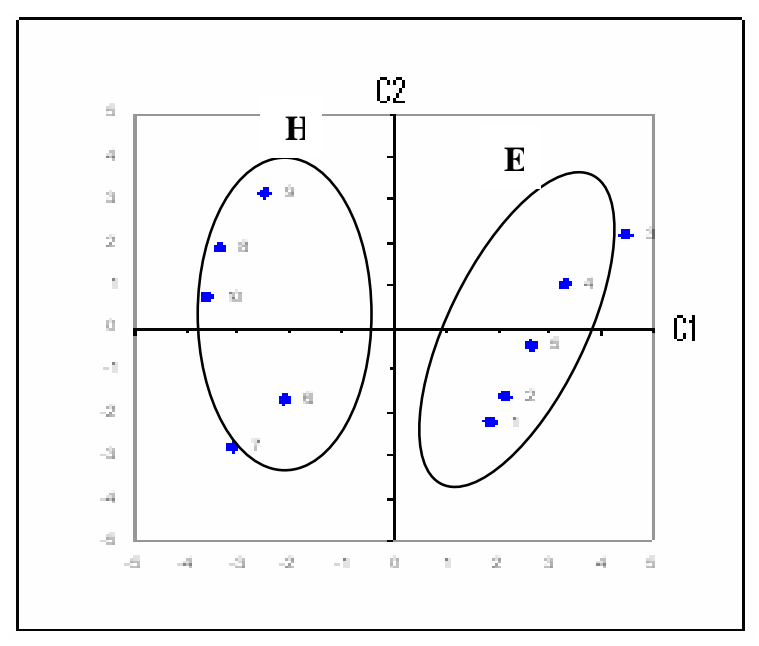

Figure 4 : Carte factorielle de distribution des relevées sur le plan factoriel $\mathrm{C} 1 \times \mathrm{C} 2$

Comme pour le plan $\mathrm{C} 1 \times \mathrm{C} 2$, le plan $\mathrm{C} 1 \times \mathrm{C} 3$ montre deux groupements de relevés: un premier groupement noté $\mathrm{H}^{\prime}$ rassemblant des relevés hivernaux et un autre E' des relevés estivaux. Notons également qu'en tenant compte la signification physicochimique et métallique de l'axe C3, les relevés numéro 6 et 8 se montrent très différant vis-à-vis de la valeur du pH et des teneurs en $\mathrm{CO}_{2}, \mathrm{SO}_{4}^{2-}, \mathrm{NO}_{2}{ }^{-}$et $\mathrm{Fe}^{2+}$ (Figure 5).

Concernant $\mathrm{Ca}^{2+}$ et $\mathrm{Mg}^{2+}$, les résultats ont montré des différences de concentration entre les relevés estivaux et ceux hivernaux. Ce phénomène semble être issu des variations climatiques du milieu. En période chaude l'action de l'évaporation combinée à la haute température favorise une dissolution plus importante de la roche mère [10], d'où les teneurs élevés en $\mathrm{Ca}^{2+}$ et $\mathrm{Mg}^{2+}$ de certains relevés de l'été.

Ceux numérotés 1,2 et 3 pour le $\mathrm{Mg}^{2+}$ et ceux numérotés 2, 4 et 5 pour le $\mathrm{Ca}^{2+}$ en sont des exemples. 


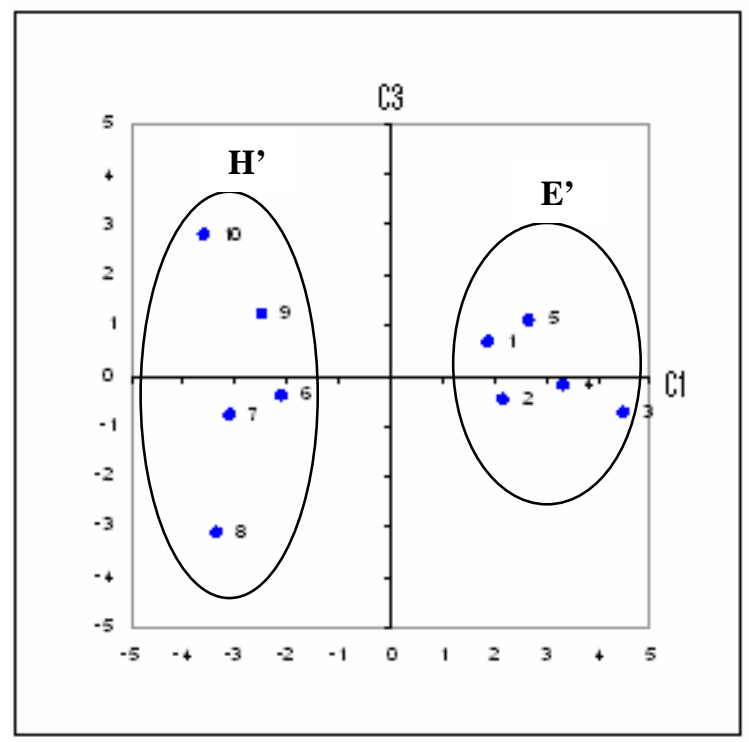

Figure 5 : Carte factorielle de distribution des relevées sur le plan factoriel $\mathrm{C} 1 \times \mathrm{C} 3$

Pour le fer, ce sont les relevés de l'hiver qui ont présenté des teneurs plus élevés. Le drainage et le lessivage du bassin versant, notamment les montagnes de la Guinée (connus par leur richesse en fer) par les eaux de pluie de ce période semblent être une cause.

Pour l'Al ${ }^{2+}$ et $\mathrm{Sr}^{2+}$, à l'exception des relevés numéros 3 et 4 , les concentrations notées ne sont trop élevées et ne semblent pas être influencées par les variations saisonnières. Les teneurs relativement élevées (3 et 4) sont effectués respectivement en SD, connue par son activité domestique ou en SRv qui reçoit des rejets industriels de la sucrerie C.S.S. Ainsi, nous notons la pollution métallique de ces deux stations.

Signalons, en outre, que l'analyse des données par l'ACP a montré que cinq éléments évalués ( $\mathrm{T}, \mathrm{MES}, \mathrm{Cl}, \mathrm{PO}_{4}{ }^{2-}$ et $\mathrm{HCO}_{3}{ }^{-}$) n'entrent pas d'une manière significative dans la typologie physico-chimique du milieu. Cependant, il noter que pour le $\mathrm{HCO}_{3}$ - les teneurs enregistrées dans le milieu sont toutes relativement élevées mais peu variables.

Par ailleurs, les valeurs de $\mathrm{K}^{+}, \mathrm{NO}_{3}{ }^{-}$et $\mathrm{SO}_{4}{ }^{2-}$ les plus élevées sont présentées par le relevé numéro 8 , effectué dans la station SD qui est 
connue par une importante activité domestique (lavage des vêtements et des vaisselles, natation, rejets des eaux usées, etc.) ce qui semble être une cause principale. SD est donc la station la plus polluée en substances organiques.

\section{Conclusion}

Les variables physico-chimiques ne participent pas toutes à la typologie des eaux de la partie étudiée du Fleuve Sénégal, au niveau de Rosso en Mauritanie. Parmi les éléments physicochimiques que nous avons évalué seules 14 participent d'une manière non négligeable. Pour plusieurs variables l'action de l'influence de la saisonnalité est très nette. En effet, les résultats montrent une répartition en deux groupements de relevés: un groupement formé de relevés estivaux et un autre groupement de relevés hivernaux et dont les caractéristiques physicochimiques sont opposées. Le premier est caractérisé par des concertations élevés en $\mathrm{Ca}^{2+}, \mathrm{Mg}^{2+}, \mathrm{Na}^{+}, \mathrm{Zn}^{2+}$ et faibles en $\mathrm{NO}_{3}{ }^{-}$et $\mathrm{K}^{+}$, ce qui n'est le cas du second groupe.

Pour les teneurs de l'Al ${ }^{3+}$ et le $\mathrm{Sr}^{2+}$, l'influence du facteur "saison" n'est pas notable. Vis-à-vis du pH et des teneurs en $\mathrm{CO}_{2}, \mathrm{SO}_{4}{ }^{2-}, \mathrm{NO}_{2}$ - et $\mathrm{Fe}^{2+}$, seuls des relevés 6 et 8 ont montré des caractéristiques opposées. Les autres sont plus ou moins homogènes.

Une pollution d'origine organique s'est enregistrée dans les stations SD et d'origine organique et métallique en SRv. La première station est connue par une importante activité humaine alors que la deuxième reçoit des rejets d'eau usée de la sucrerie C.S.S. Cependant, malgré cette pollution, les teneurs de la majorité des éléments évalués ne sont pas menaçants pour l'irrigation. 


\section{Références}

[1] - Mohamed OUKHOUYA, Etude de la valorisation agricole de deux composts d'ordures ménagères. Mémoire de troisième cycle, option sciences du sol, I.A.V HASSANII, (1999) p 26.

[2] - Abddayem MaawiateContribution à l'étude de l'impact de l'irrigation sur la qualité des eaux et des sols dans le Delta du fleuve Sénégal, Cas du périmètre de M'Pourié (Mauritanie), Mémoire de troisième cycle en Génie Rural, Option: Irrigation, IAV II, Rabat, Maroc,(1997): 258p.

[3] - Mohamed Ould Sid'Ahmed Ould KANKOU Vulnérabilité des eaux et des sols de la rive droite du fleuve Sénégal en Mauritanie. Thèse de Doctorat, l'université de limoges, option Chimie et Microbiologie de l'Eau, France (2004) 239p

[4] - M. Halima AMRANI Mémoire de fin d'étude pour l'obtention du diplôme d'Ingénieur d'Etat en Agronomie. "Revue bibliographie sur la pollution sur l'oued Sebou " I.A.V Hassan (2000) 3 -II.

[5] - Boschet A.F., - Ressources en eau et santé en Europe- Journal Européen d'Hydrologie, (2002) 1- 39.

[6] - J. Rodier L'analyse de l'eau, eaux naturelles, eau résiduaires, eau de mer, 7 e édition Paris (1984):67 P.

[7]- Ministère de l'Economie des Finances et de l'industrie, Représentations graphiques des résultats d'analyses eau minérale (Note technique $n^{\circ}$ 8) (1996)3.P

[8] - J. M. BOUROCHE et G. SAPORTA, , l'Analyse des données, que sais-je, Presses universitaires de France, (1980)127p.

[9] - J. P. BENZECCRI et coll., L'analyse des données, I : La taxinomie, t. II : L'analyse des correspondances, Dunod, 3e éd. (1979)

[10] - Jadal M. Pollution chimique, métallique et microbiologique de L'estuaire de L'oued oum-Er-rdia: Suivi de la micropollution et Ses incidences sur les différentes composantes de cet écosystème. Thèse de Doctorat d'état ES-Science biologique, université Ibn Tofail Kenitra, Maroc, : 65 (2002) 230 